PROCEEDINGS OF THE

AMERICAN MATHEMATICAL SOCIETY

Volume 131, Number 5, Pages 1623-1627

S 0002-9939(02)06770-9

Article electronically published on October 15, 2002

\title{
DIAGRAMS OF DIVIDE LINKS
}

\author{
SERGEI CHMUTOV
}

(Communicated by Ronald A. Fintushel)

\begin{abstract}
Recently N. A'Campo suggested a construction of a link from a generic immersion of a curve into a 2-disk. It is tightly related to the singularity theory. In this paper, we give a simple procedure to draw a diagram of the link from a picture of the curve.
\end{abstract}

There are several fascinating relations of plane immersed curves and links. One of them which goes through Legendrian links led Arnold [5] to the discovery of three simple invariants $J^{+}, J^{-}, S t$ of such a curve. N. A'Campo in [2] suggested another construction of a link from a generic immersion of a curve into a 2-disk. It is tightly related to the singularity theory. If $f_{\mathbb{R}}:\left(\mathbb{R}^{2}, 0\right) \rightarrow(\mathbb{R}, 0)$ is a germ of an analytic function whose complexification $f_{\mathbb{C}}$ has an isolated critical point, then one can define a link $\mathcal{L}$ of the singularity $f_{\mathbb{C}}$ as an intersection of the zero-level variety of $f_{\mathbb{C}}$ with a small 3 -sphere in $\mathbb{C}^{2}$ centered at the critical point. Such links are sometimes called algebraic links. On the other hand, in singularity theory it is useful [1, 9] (see also [6]) to consider a small perturbation $D$ of the real plane singular curve $\left\{f_{\mathbb{R}}=0\right\}$ which is a generic immersed curve with the maximal possible number of double points. A'Campo 2 restored the link $\mathcal{L}$ directly from the curve $D$.

In this paper we give a simple procedure to draw a diagram of the link $\mathcal{L}$ from a picture of $D$ (Theorem 2.2). It is essentially a particular case of the results of 8. An advantage of our approach is that we obtain a link diagram directly from a divide picture without deforming it into a so-called ordered Morse signed divide as in [8]. A similar method to draw diagrams was found by M. Hirasawa in [10. Our diagrams are obviously symmetrical in a sense that the rotation of $\mathbb{R}^{3}$ by $180^{\circ}$ around the $x$-axis reverses the orientation of $\mathcal{L}$.

\section{DIVIDES AND THEIR LINKS}

Definition $1.1([2,3]) . \quad A$ divide $D$ is the image of a generic immersion of a finite number of copies of the unit interval $I=[0,1]$ in the unit disk $B \subset \mathbb{R}^{2}$ such that $\partial I$ is embedded in $\partial B$ and double points are the only singularities allowed.

We consider divides up to isotopy of the disk $B$. The isotopy does not assume to be identical on the boundary $\partial B$.

Received by the editors November 19, 2001 and, in revised form, December 17, 2001.

2000 Mathematics Subject Classification. Primary 57M25.

Key words and phrases. Links, fibered links, link diagram, divide.

(C)2002 American Mathematical Society 
Example 1.2. The curve $x^{3}+y^{4}=0$ has a singularity of type $E_{6}$ at the origin [6]. A small perturbation of it is a divide which looks as follows.

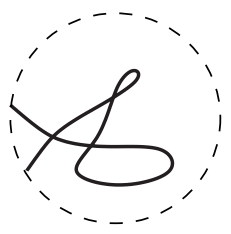

Definition $1.3([2,[3])$. Let $x$ be the horizontal coordinate on the disk $B$ and $y$ be the vertical coordinate. $A$ divide link $\mathcal{L}_{D}$ is a link in the 3 -sphere $S^{3}=$ $\left\{(x, y, u, v) \in \mathbb{R}^{4} \mid x^{2}+y^{2}+u^{2}+v^{2}=1\right\}$ such that $(x, y)$ is a point on $D$ while $u$ and $v$ are the coordinates of a tangent vector to $D$ at the point $(x, y)$.

Therefore each interior point of $D$ has two corresponding points on $\mathcal{L}_{D}$, and a boundary point of $D$ gives a single point on $\mathcal{L}_{D}$.

The link $\mathcal{L}_{D}$ has a natural orientation. Indeed, choose any orientation of every branch of $D$. Let $(u, v)$ be the tangent vector to $D$ at $(x, y)$ pointing to the direction of the chosen orientation of $D$. Then the orientation of $\mathcal{L}_{D}$ is given by the vector $(\dot{x}, \dot{y}, \dot{u}, \dot{v})$. It is easy to see that this orientation of $\mathcal{L}_{D}$ does not depend on the choice of orientations of branches of $D$.

The number of components of $\mathcal{L}_{D}$ equals to the number of branches of the divide $D$ which is the number of the copies of the unit interval $I$ in Definition 1.1. In particular, if $D$ consists of only one branch as in Example 1.2 then $\mathcal{L}_{D}$ will be a knot.

Remark 1.4. Topological type of a divide link does not change under a regular transversal isotopy of the disk $B$. Therefore it does not depend on the choice of coordinates in Definition 1.3. Also it does not change under a moving of a piece of the curve $D$ through a triple point 8 . In particular, the following two divides have the same knot type as the one in Example 1.2:
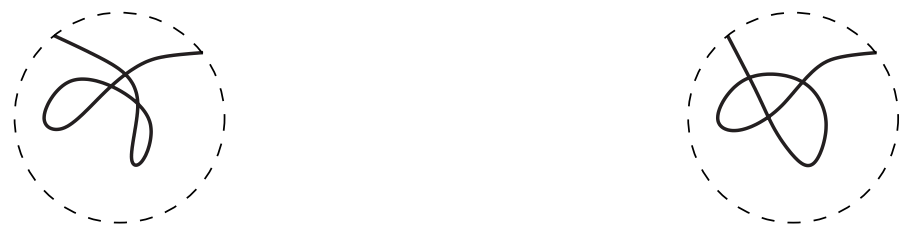

Remark 1.5. In 2] A'Campo proved that all algebraic links are divide links. In 3 he showed that the links $\mathcal{L}_{D}$ corresponding to a connected divide $D$ are fibered, and computed their monodromy in terms of the combinatorics of divide $D$. Moreover, he proved that the unknotting number of a one-branch divide knot $\mathcal{L}_{D}$ equals the number of double points of $D$. Not all fibered links have the form $\mathcal{L}_{D}$. Figure eight knot $4_{1}$ is not a divide knot. It is not clear how large is the class of divide links in the class of all fibered links.

Remark 1.6. It has been known for a long time 7] that algebraic knots are classified by the Alexander polynomial. N. A'Campo (4]) found two different divide knots with the same Alexander polynomial. 


\section{DiAgrams OF DIVIDE LINKS}

Definition 2.1. Let us call a divide generic if its points with vertical tangents differ from the double points and the boundary points and their $x$-coordinates are pairwise different. The divide in Example 1.2 is generic. Any divide can be made generic by a small deformation.

Theorem 2.2. For a generic divide $D$ a link diagram of $\mathcal{L}_{D}$ can be drawn in the following way:

(1) Consider a horizontal line below the disk B, say the line $\{y=-1.5\}$. Let $s$ be the symmetry with respect to the line. We are going to draw the link diagram of $\mathcal{L}_{D}$ by modifications of the union $D \cup s(D)$.

(2) Replace each double point of the union by a crossing of the type $>$.

(3) Connect each boundary point $p$ of $D$ with $s(p)$ by a vertical string.

(4) Replace a small piece of our curve near each point $p$ with a vertical tangent by two vertical strings connecting the upper and lower parts of the picture as in the examples below. The strings make a positive half-twist at the line of the reflection s. Note that at the upper part of the picture the right string goes below all intersected intervals of $D$ while the left string goes above the intervals. Correspondingly at the lower part of the picture the right string goes above the intervals of $s(D)$ and the left string goes below the intervals. Examples [2.3 and 2.4 demonstrate this.

Example 2.3. For the divide of Example1.2 the theorem gives the diagram shown in Figure 1.

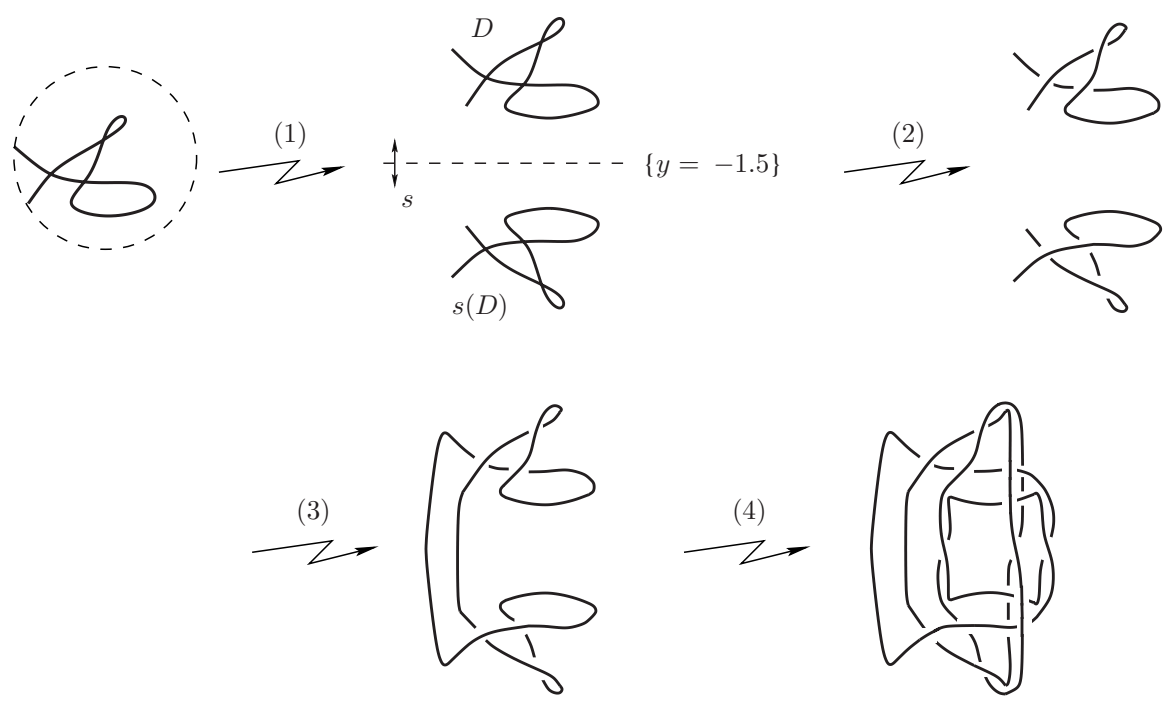

Figure 1. 
Example 2.4. We borrowed this divide from [3]. The corresponding knot is $10_{145}$.
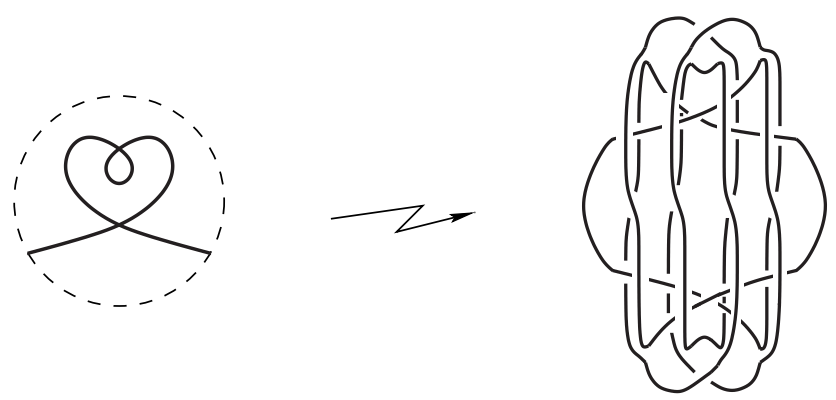

Remark 2.5. As it was noted in 8 the theorem is also valid in the situation when we allow closed immersed components in the definition of a divide.

2.1. Proof of the Theorem [2.2. The theorem follows from the results [8] which give a representative braid for an ordered Morse signed divide (OMS). An OMS is a divide $D$ such that the $x$-coordinate as a function on $D$ has only two critical values: $a$ as the minimum critical value and $b$ as the maximum critical value; and the $x$-coordinate of each double point is between $a$ and $b$. Besides this a sign + or - is attached to each double point of $D$. See the details in $[8$. An OMS is not a generic divide in our sense. Also in [8] there is an algorithm transforming an arbitrary divide to OMS (and attaching signs to double points). We use such a particular transformation pulling down a narrow tail near each critical point of the function $\left.x\right|_{D}$ and then move a minimum (resp. maximum) left (resp. right) to the level $a$ (resp. $b$ ). For the divide of Example 2.4 this transformation gives the following OMS:
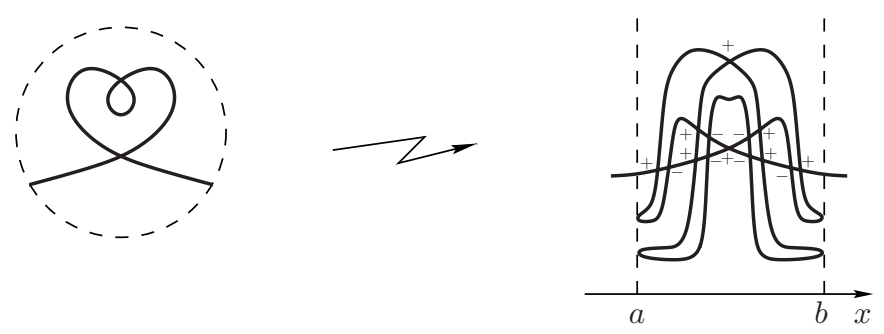

Then applying Proposition 4.2 from [8] we get the following representative braid:

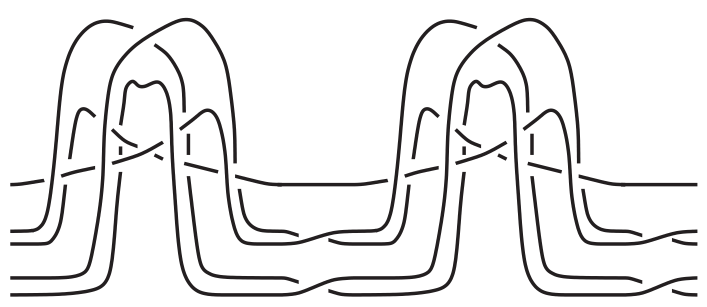

The closure of this braid gives a knot diagram isotopic to ours. It is clear that the same arguments work for any generic divide as well. 


\section{ACKNOWLEDGMENTS}

I express my deep gratitude to the Department of Mathematics at Mount Holyoke College where this work was done, for their warm hospitality and stimulating atmosphere. I thank N. A'Campo, M. Hirasawa and the referee for valuable comments.

\section{REFERENCES}

1. N. A'Campo, Le groupe de monodromie du déploiement des singularités isolées de courbes planes I, Math. Ann., 213 (1975) 1-32. MR 51:13282

2. N. A'Campo, Real deformations and complex topology of plane curve singularities, Ann. Fac. Sci. Toulouse Math. (6) 8 (1999), no. 1, 5-23 (see also alg-geom/9710023). MR 2001d:32043a

3. N. A'Campo, Generic immersions of curves, knots, monodromy and gordian number, Inst. Hautes Etudes Sci. Publ. Math. No. 88 (1998), 151-169 (1999) (see also math.GT/9803081). MR 2001b:57009

4. N. A'Campo, Private communication, January, 2001.

5. V. I. Arnold, Topological invariants of plane curves and caustics, University Lecture Series, Vol. 5, AMS, Providence, RI, 1994. MR 95h:57003

6. V. I. Arnold, S. M. Gusein-Zade, A. N. Varchenko, Singularities of differentiable maps, Vol. II, Birkhäuser, Boston, MA, 1988. MR 89g:58024

7. W. Burau, Kennzeichnung der Schlauchknoten, Abh. Math. Sem. Univ. Hamburg, 9 (1932) $125-133$.

8. O. Couture, B. Perron, Representative braids for links associated to plane immersed curves, Journal of Knot Theory and Its Ramifications, 9 (2000) 1-30. MR 2001b:57012

9. S. M. Gusein-Zade, Intersection matrices for certain singularities of functions of two variables, Functional Anal. and its Appl., 8 (1974) 11-15. MR 49:3202

10. M. Hirasawa, Visualization of A'Campo's fibered links and unknotting operations, to appear in Topology and its Applications.

Department of Mathematics, The Ohio State University at Mansfield, 1680 UniverSity Drive, Mansfield, Ohio 44906

E-mail address: chmutov@math.ohio-state.edu 